\title{
Method for measurement of the angles of a 45-deg deflecting (half penta) prism
}

\author{
S. Madhusudana Rao \\ K. Narasimha Rao \\ Indian Institute of Science \\ Department of Instrumentation \\ Bangalore 560 012, India
}

\begin{abstract}
A simple technique is devised to measure the angles of $45-$ deg deflecting (half penta) prisms, without using the expensive spectrometers, autocollimators, and angle gauges. () 2000 Society of PhotoOptical Instrumentation Engineers. [S0091-3286(00)03506-6]

Subject terms: prisms; metrology.

Paper 980439 received Nov. 23, 1998; revised manuscript received Nov. 17, 1999; accepted for publication Feb. 16, 2000.
\end{abstract}

\section{Introduction}

The usual practice of comparing the angles of a given prism against a standard angle gauge (or a gauge combination) by using an angle dekkor (autocollimator) is the simplest and most accurate among the several methods used for testing the angles of prisms. ${ }^{1-5}$ In this method a suitable angle gauge (or gauge combination) is essential for conducting the experiment. Alternate methods are suggested in Refs. 6 to 10 for the circumstances where an autocollimator and angle gauges are not available. In these methods, the angular deviation from symmetry of two surfaces with respect to a side as baseline is determined from the measurements of the screen distance and the separation of the reflected laser spots on a screen for two positions before and after the rotation of a partially or fully polished optical component by $180 \mathrm{deg}$.

Since detailed description of the experiments is already given in previous papers, ${ }^{6-10}$ here we present only a brief outline of the method for the measurement of the angles of a 45-deg deflecting prism.

\section{Principles of the Method}

The solid lines of Fig. 1 depict the 45-deg deflecting (half penta) prism. For the purpose of theoretical discussion some of the sides are extended by broken lines to form triangles. The sides of the prism are represented by the symbols $S_{1}, S_{2}, S_{3}$, and $S_{4}$, and the angles by $A_{1}, A_{2}$, $A_{3}$, and $A_{4}$. In most common uses of the prism it is not necessary for the angle $A_{1}$ to be a right angle or for the surface $S_{1}$ to be polished. However, for making an initial standard, this method requires a slightly larger prism with all polished sides and a 90-deg angle for $A_{1}$. For an ideal prism $A_{1}=90 \mathrm{deg}, A_{2}=A_{3}=112.5 \mathrm{deg}, A_{4}=45 \mathrm{deg}$, and the corresponding angles $X=45 \mathrm{deg}$ and $Y=22.5 \mathrm{deg}$. Let the angles of an approximate prism be $A_{1}=90+\alpha_{1}, A_{2}$ $=112.5+\alpha_{2}, A_{3}=112.5+\alpha_{3}$, and $A_{4}=45+\alpha_{4}$, and correspondingly $X=45+\beta$ and $Y=22.5+\gamma$, where $\alpha_{1}, \alpha_{2}$, $\alpha_{3}, \alpha_{4}, \beta$, and $\gamma$ are the errors of the angles $A_{1}, A_{2}, A_{3}$, $A_{4}, X$, and $Y$. The experimentally determined values for $\alpha_{1}, \alpha_{2}, \alpha_{3}, \alpha_{4}, \beta$, and $\gamma$ can either be positive or negative.

\subsection{Right Angle (90 deg)}

Figure 2 illustrates the principle of measurement of the 90deg angle of a prism. The detailed description of the experiments can be found in Refs. 6-10. The error in the 90-deg angle is given by

$\alpha_{1}=\frac{A B}{4 O C}$.

\subsection{Other Angles (45, 112.5, and $22.5 \mathrm{deg})$}

Figure 3 illustrates the second part of the experiment. The prism surface $S_{3}$ is placed on the optical flat. The angular deviation from symmetry ( $\left.\theta_{1}\right)$ of the surfaces $S_{4}$ and $S_{1}$ with respect to baseline $S_{3}$ (Fig. 1) is given by

$\theta_{1}=\frac{L M}{4 O N}$

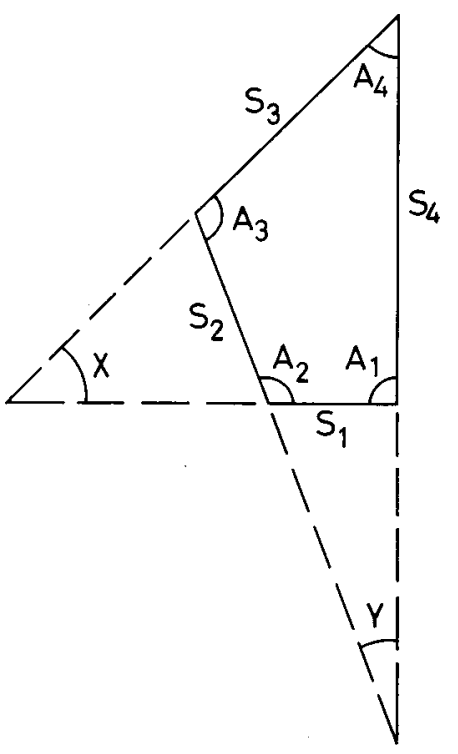

Fig. 1 Schematic diagram of a 45-deg deflecting prism with 22.5deg corner removed (i.e., a half penta prism). For the purpose of theoretical discussion some of its sides are extended by the broken lines to form triangles. 


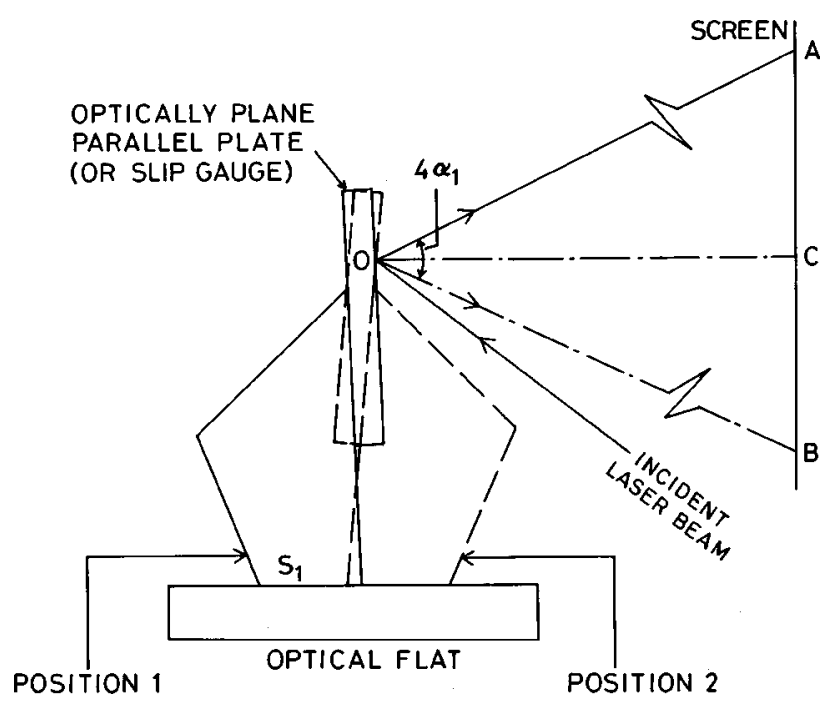

Fig. 2 Schematic diagram showing the principle of testing the 90deg angle (first part of the experiment). The angle $4 \alpha_{1}$ is greatly exaggerated in this diagram.

Therefore, from Ref. 10,

$X=45-\frac{\alpha_{1}}{2} \pm \theta_{1}$,

$A_{4}=45-\frac{\alpha_{1}}{2} \mp \theta_{1}$.

The errors of the angles $X$ and $A_{4}$ are given by

$\beta=-\frac{\alpha_{1}}{2} \pm \theta_{1}$,

$\alpha_{4}=-\frac{\alpha_{1}}{2} \mp \theta_{1}$.

Figure 4 illustrates the third part of the experiment. The experiment is conducted, as above for the surfaces $S_{1}$ and $S_{3}$, by placing $S_{2}$ on the optical flat. We now have by the preceding discussion

$\theta_{2}=\frac{P Q}{4 O R}$,

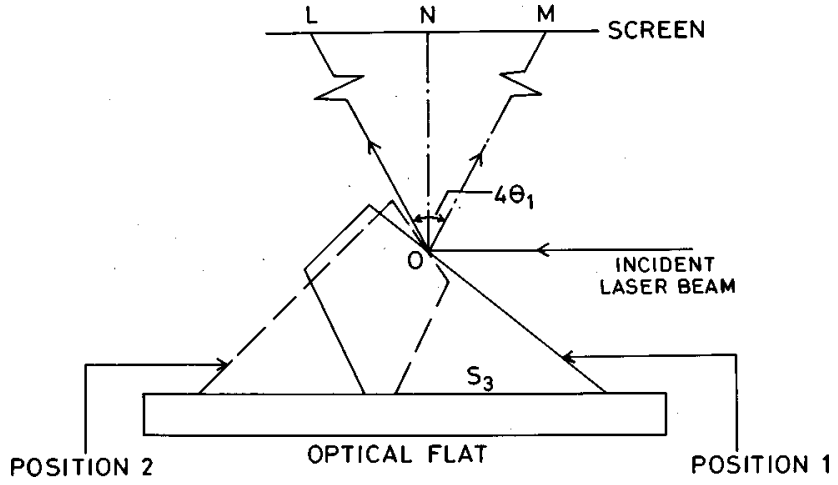

Fig. 3 Schematic diagram showing the principle of testing the 45deg angles $A_{4}$ and $X$ of Fig. 1 (second part of the experiment). The angle $4 \theta_{1}$ is greatly exaggerated in this diagram.

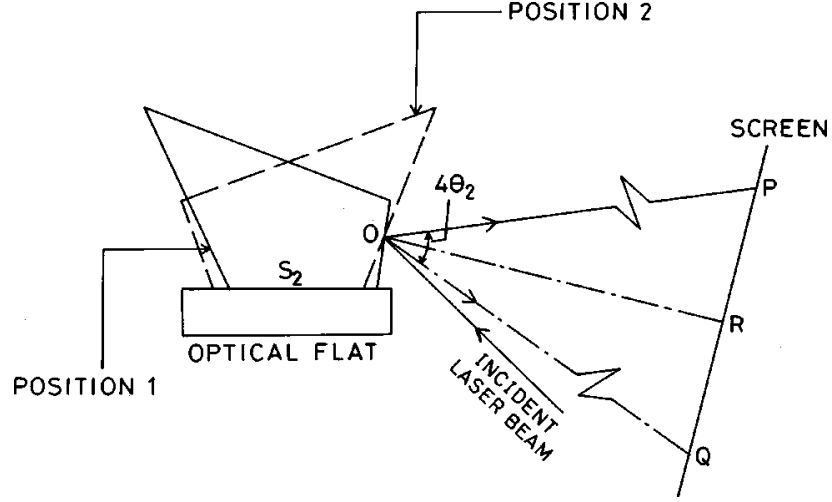

Fig. 4 Schematic diagram showing the principle of testing the 112.5-deg angles (third part of the experiment). The angle $4 \theta_{2}$ is greatly exaggerated in this diagram.

$\alpha_{2}=\frac{\beta}{2} \mp \theta_{2}$,

$\alpha_{3}=\frac{\beta}{2} \pm \theta_{2}$.

The error $\gamma$ of the angle $Y$ (Fig. 1) is given by $\gamma=-\left(\alpha_{3}+\alpha_{4}\right)$.

The details about the measurement of the angles $\alpha_{1}, \theta_{1}$, $\theta_{2}$, etc., can be found in Refs. 6-10.

Experiments are conducted to measure the angles by the proposed method and also by using a micrometer angle dekkor (autocollimator). The results are given in Table 1.

The angular deviations from symmetry $\left(\alpha_{1}, \theta_{1}\right.$, and $\theta_{2}$ ) can also be measured by using an autocollimator instead of using a laser beam. The only difference is that we get $2 \alpha_{1}, 2 \theta_{1}$, and $2 \theta_{2}$ from the autocollimator rather than $4 \alpha_{1}, 4 \theta_{1}$, and $4 \theta_{2}$ as shown in Figs. 2, 3, and 4 (since the autocollimator graticules and micrometer screws are designed to read half of the actual angles). Thus the technique can also be extended for carrying out measurements with an autocollimator when a suitable angle-gauge combination is not available.

The accuracy of the measurements depends upon the distances $(O C, O N$, and $O R$ of Figs. 2, 3, and 4) of the screen from the point of incidence of the laser beam on the optical component. For this case $O C=O N=O R=5 \mathrm{~m}$. These distances are measured to an accuracy of $1 \mathrm{~mm}$. A

Table 1 Experimental results for a 45-deg deflecting (half penta) prism.

\begin{tabular}{ccc}
\hline \hline & \multicolumn{2}{c}{ Error (arcsec) } \\
\cline { 2 - 3 } $\begin{array}{c}\text { Name of } \\
\text { the angle }\end{array}$ & $\begin{array}{c}\text { By the proposed } \\
\text { method }\end{array}$ & $\begin{array}{c}\text { By autocollimator } \\
\text { and angle gauges }\end{array}$ \\
\hline$A_{1}$ & -48.16 & -48.0 \\
$A_{2}$ & 15.14 & 15.5 \\
$A_{3}$ & -9.00 & -9.5 \\
$A_{4}$ & 42.02 & 42.5 \\
$Y$ & -33.02 & -33.0 \\
\hline \hline
\end{tabular}


method of increasing the screen distance is given in Ref. 8 . The distances $A B, L M$, and $P Q$ are measured to an accuracy of $0.01 \mathrm{~mm}$.

From the error analysis (which is identical to that given in Ref. 10), the accuracy in the measurement of angles is $1 / 3$ arcsec, whereas with the micrometer angle dekkor it ranges from 0.5 to 2 arcsec, depending on the performance of the micrometer screw.

\section{Conclusions}

The proposed method does not require precision spectrometers, autocollimators, and standard angle gauges. It is a simple, accurate, and relatively inexpensive method.

\section{Acknowledgments}

The authors wish to thank Prof. S. Mohan, Prof. H. L. Bhat, and Prof. M. V. Krishnamurthy for the useful discussions while conducting these experiments.

\section{References}

1. C. Deve, Optical Workshop Principles, 2nd ed., Chap. VI, pp. $263-$ 275, Hilger \& Watts, London (1954).

2. F. Twyman, Prism and Lens Making, 2nd ed., Chap. 11, pp. 405-413, Hilger \& Watts, London (1957).

3. A. S. De Vany, Master Optical Techniques, Chaps. 6-13, pp. 73-189, John Wiley \& Sons, New York (1981).

4. D. F. Horne, Optical Production Technology, Chap. 11, pp. 343-351, Adam Hilger, London (1972).

5. B. K. Johnson, Optics and Optical Instruments, Chap. 8, pp. 159-200, Dover Publications, New York (1960)
6. M. V. R. K. Murty and R. P. Shukla, "Method for measurement of parallelism of optically parallel plates," Opt. Eng. 18(3), 352-353 (1979).

7. S. Madhusudana Rao, "Method for measurement of the angles of 90-deg, 45-deg, 45-deg and 60-deg, 30 deg, 90-deg prisms," Opt. Eng. 36(1), 198-200 (1997).

8. S. Madhusudana Rao, "Method for measurement of the angles of an equilateral (60-deg) prism," Opt. Eng. 36(5), 1508-1509 (1997).

9. S. Madhusudana Rao, "Method for measurement of the angles of polygons," Opt. Eng. 36(7), 2062-2067 (1997).

10. S. Madhusudana Rao and K. Narasimha Rao, "Method for measurement of the angles of a pentaprism,' Opt. Eng. 37(4), 1368-1371 (1998).

S. Madhusudana Rao received his MSc degree in nuclear physics from Andhra University at Waltair, India, in 1972 and began working in the field of testing and calibration of optical instruments with the Andhra Scientific Company (presently BEL) in Masulipatam. In 1977 he joined the Indian Institute of Science, Bangalore, where he received an MSc (Engg) degree in 1994. He is now a principal research scientist in the Department of Instrumentation. He has several reserach publications to his credit. His research interests include optical instrumentation, stress analysis, and image processing. He is a member of the Instrument Society of India.

K. Narasimha Rao received his MSc (Tech) degree in applied physics from Andhra University at Waltair, India, in 1971 and joined the Indian Institute of Science in Bangalore, where he received his $\mathrm{PhD}$ degree in 1988. He is now a principal research scientist in the Department of Instrumentation. He has published about 50 research papers. His research interests are thin films for a variety of applications and optical instruments. He is a member of the Instrument Society of India, the Indian Vacuum Society, and the Indian Laser Association. 